\title{
Exponential tuning of the coupling constant of coupled microcantilevers by modifying their separation
}

\author{
Eduardo Gil-Santos, Daniel Ramos, Valerio Pini, Montserrat Calleja, and Javier Tamayo ${ }^{\text {a) }}$ \\ Instituto de Microlectrónica de Madrid (IMM), CSIC, Isaac Newton 8 (PTM), Tres Cantos, \\ 28760 Madrid, Spain
}

(Received 27 December 2010; accepted 2 March 2011; published online 22 March 2011)

\begin{abstract}
Vibration localization in coupled nanomechanical resonators has emerged as a promising concept for ultrasensitive mass sensing. It possesses intrinsic common mode rejection and the mass sensitivity can be enhanced with no need of extreme miniaturization of the devices. In this work, we have experimentally studied the role of the separation between cantilevers that are elastically coupled by an overhang. The results show that the coupling constant exponentially decays with the separation. In consistency with the theoretical expectations, the mass sensitivity is inversely proportional to the coupling constant. Finite element simulations show that the coupling constant can be exponentially reduced by increasing the ratio of the cantilever separation to the overhang length. (C) 2011 American Institute of Physics. [doi:10.1063/1.3569588]
\end{abstract}

Nanomechanical resonators such as vibrating cantilevers, doubly clamped beams, and trampolines to name a few geometries have been proposed for ultrasensitive mass sensing. ${ }^{1-5}$ The principle is that molecular adsorption on the surface of the resonator gives rise to an increase in the active mass of the resonator that makes the resonance frequency to decrease. The continuous advancements in top-down microand nanofabrication techniques has made possible increasingly smaller nanomechanical resonators with detection limits in the zeptogram range $\left(10^{-21} \mathrm{~g}\right) .^{6}$ Moreover, resonant nanowires and nanotubes fabricated by bottom-up methods are on the verge of single atom resolution $\left(1.6610^{-24} \mathrm{~g}\right)^{7,8}$ The main disadvantage in the fabrication of extremely small nanomechanical resonators is that the deviations and imperfections inherent to the fabrication at nanoscale makes difficult to obtain reproducible mechanical properties and responses to molecular adsorption. In addition, very large scale integration of these resonators is still technologically complex.

An approach that is no longer depending on the extreme miniaturization of the devices is the use of coupled nanomechanical resonators fabricated by standard silicon technology. ${ }^{9-14}$ When the resonators are identical, the vibration of the eigenmodes is delocalized over the array. In a similar way to the Anderson's localization, the addition of the mass on one of the resonators leads to the spatial localization of the eigenmodes. ${ }^{9,10,12}$ Since vibration localization is insensitive to uniform adsorption, coupled nanomechanical resonators allows decoupling of unspecific and specific molecular adsorption in differentially sensitized resonators. Theoretically, the sensitivity of these devices could be enhanced by reducing the mechanical coupling constant with no need of miniaturization of the nanomechanical resonators. $^{12}$

Here we have fabricated coupled mechanical systems consisting of two silicon nitride microcantilevers elastically coupled by an overhang at their base. The aim is to study the dependence of the mass sensitivity on the coupling constant

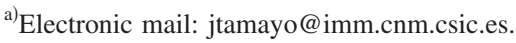

that in this work is tuned by changing the separation between cantilevers.

An optical micrograph of one of the fabricated devices is shown in Fig. 1(a). The cantilever length $(L)$, width $(W)$, and thickness were $25 \mu \mathrm{m}, 10 \mu \mathrm{m}$, and $0.1 \mu \mathrm{m}$, respectively. The overhang length $(b)$ was fixed to $8 \mu \mathrm{m}$, whereas the gap $(p)$ between cantilevers was varied from 2 to $100 \mu \mathrm{m}$. The
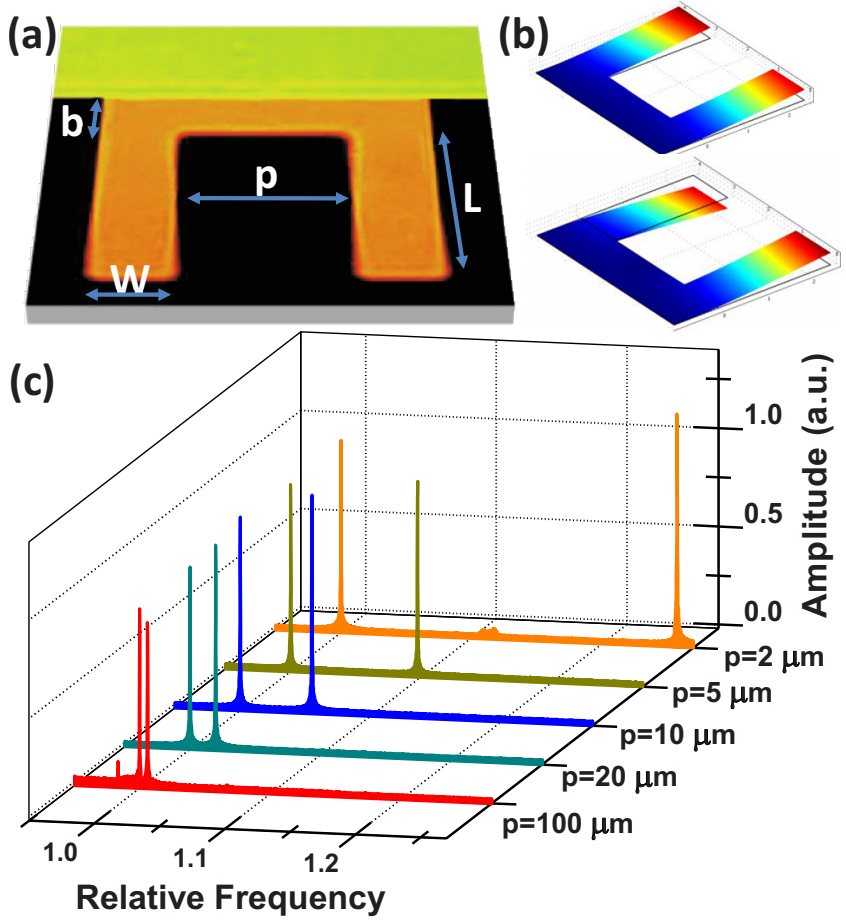

FIG. 1. (Color online) (a) Optical micrograph of a system of coupled cantilevers connected by an overhang. The cantilevers were fabricated in low stress silicon nitride. The length, $L$, width, $W$, and thickness of the cantilevers were $25 \mu \mathrm{m}, 10 \mu \mathrm{m}$, and $0.1 \mu \mathrm{m}$, respectively. The overhang length, $b$, was $8 \mu \mathrm{m}$. In this case, the gap separation, $p$, between cantilevers was $20 \mu \mathrm{m}$. (b) Graphical depiction of the symmetric (top) and antisymmetric (bottom) vibration modes of the coupled cantilevers. (c) Evolution of the frequency spectra of the thermomechanical fluctuations of the coupled cantilevers as the gap between cantilevers is reduced from 100 to $2 \mu \mathrm{m}$. The frequency axis is normalized to the resonance frequency of the symmetric mode (first peak) that is about $135 \mathrm{kHz}$. 


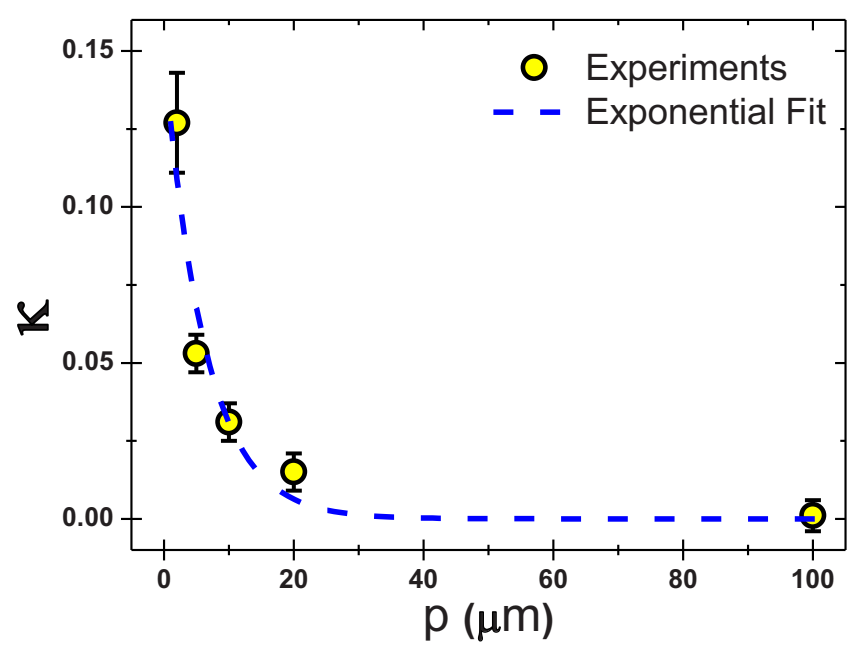

FIG. 2. (Color online) Coupling constant vs the gap separation between coupled cantilevers (symbols). The coupling constant is calculated from the difference between the antisymmetric and symmetric eigenfrequencies. The dashed line is the fitting of the data to an exponential curve.

stochastic vibration of the cantilevers was measured in high vacuum at $10^{-7}$ mbar by a home-made Michelson interferometer that uses a $\mathrm{He}-\mathrm{Ne}$ laser $(5 \mathrm{~mW}, 633 \mathrm{~nm})$. The mechanical quality factor of these cantilevers was around 3000. The nanomechanical device can be modeled as a lumped parameter system consisting of two identical springs with elastic constant $k$ and active mass $m$ that represents the cantilevers that are connected by a third spring with constant $k_{c}$ that couples the motion of the two cantilevers and represents the overhang. This system exhibits two collective eigenmodes [Fig. 1(b)], a symmetric mode in which the cantilevers vibrate in-phase at angular resonance frequency $\omega_{s}$ and a antisymmetric mode in which the cantilevers vibrate in antiphase at a higher eigenfrequency $\omega_{a}{ }^{9,12}$ The difference between the two eigenfrequencies is inversely proportional to the stiffness of the coupling element, in our case the overhang. Therefore the coupling constant defined as $\kappa=k_{c} / k$ can be obtained from the eigenfrequencies measurement by,

$$
\kappa=\frac{1}{2} \frac{\omega_{a}^{2}-\omega_{s}^{2}}{\omega_{s}^{2}} .
$$

Notice that Eq. (1) is valid in the limit of small difference in mass of the two coupled cantilevers. Figure 1(c) shows the frequency spectra of the Brownian fluctuations of the coupled cantilevers as a function of the gap distance between the two cantilevers. We have measured the thermomechanical fluctuations, instead of externally driven vibrations because the thermal forces are spatially uncorrelated, which allows to observe, in addition to the symmetric mode, the antisymmetric mode. ${ }^{12}$ This mode is hard to detect in identical cantilevers by using for instance, piezoelectric excitation, because the driving force is in-phase on the two cantilevers. ${ }^{15}$ The frequency spectra show that as the separation between the two cantilevers increases the difference between the antisymmetric and symmetric eigenfrequencies decreases. Figure 2 shows the estimation of $\kappa$ through Eq. (1), as a function of the gap distance (symbols). We observe an exponential decay (dashed line) when the distance between cantilevers is increased. Each point of the graph corresponds with the measurement of four to five different devices. The error bars arise from fabrication deviations, in particular in

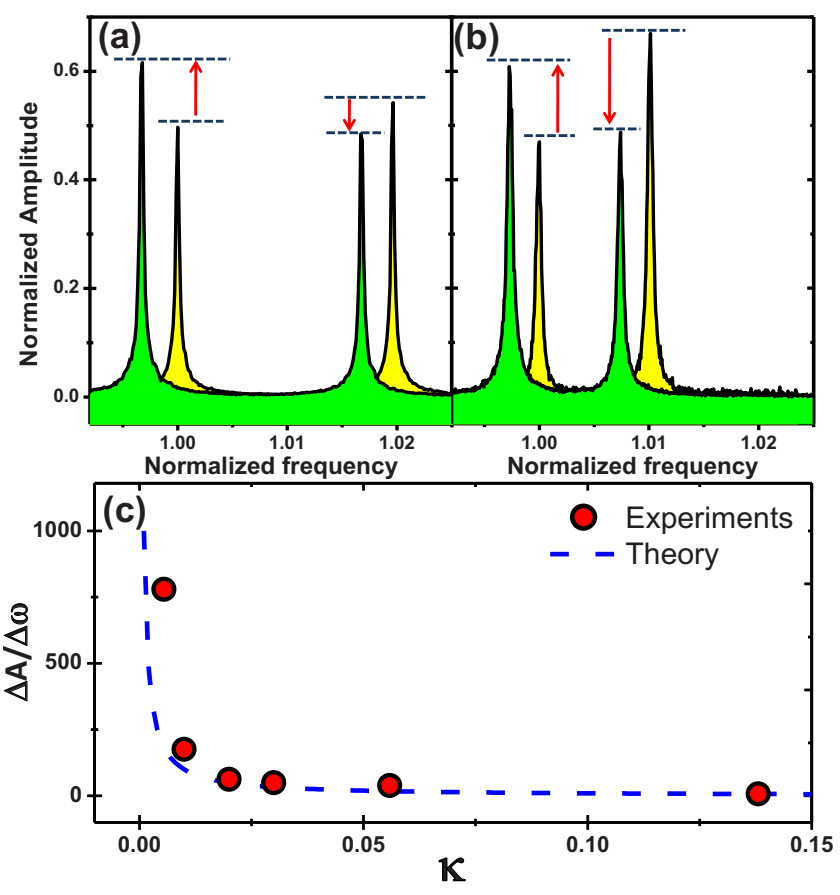

FIG. 3. (Color online) Thermal vibration spectra of coupled cantilevers before, light gray (yellow online) and after the deposition, dark gray (green online) the deposition of $100 \pm 10 \mathrm{fg}$ on one of the cantilevers for a separation gap of 10 (a) and $20 \mu \mathrm{m}$ (b). The vibration was measured on the cantilever in which the mass was added. The decrease in the eigenfrequencies is of about $0.3 \%$ in both cases, whereas the ratio between the antisymmetric and symmetric amplitude approximately decreases $30 \%$ and $60 \%$ for the gap of $10 \mu \mathrm{m}$ and $20 \mu \mathrm{m}$, respectively. (c) Ratio between the relative changes in the antisymmetric/symmetric amplitude ratio and resonance frequency shift vs the coupling constant. The symbols are experimental data and the dashed red line is a theoretical prediction based on coupled harmonic oscillator theory and the fluctuation-dissipation theorem.

the overhang length. The minimum coupling between cantilevers, $\kappa \cong 0.006$, was found for the case in which the gap is $100 \mu \mathrm{m}$.

To illustrate the effect of the coupling constant on the mass sensitivity we show the frequency spectra for two coupled systems in which the gap between cantilevers is 10 and $20 \mu \mathrm{m}$ before and after the addition of $100 \pm 10 \mathrm{fg}$ on the tip of one of the cantilevers [Figs. 3(a) and 3(b)]. The measurement was performed by measuring the vibration of the cantilever with the added mass. The mass was deposited by focusing an e-beam of a scanning electron microscope (SEM) on the wished region of the nanomechanical system. ${ }^{8,12}$ The e-beam produces the slow deposition of an amorphous carbon layer through the dissociation of organic species present in the SEM chamber. The added mass brings about a major localization of the vibration of the symmetric mode on the cantilever with the added mass. In other words, the thermal antisymmetric/symmetric amplitude ratio defined as $a_{t h}$ decreases with the mass. The opposite occurs on the cantilever where the mass was not deposited. By increasing the gap between cantilevers from 10 to $20 \mu \mathrm{m}$, the $a_{t h}$ variation increases from $30 \%$ to $60 \%$. However the change in the frequencies of the two eigenmodes remains constant about $0.3 \%$ since the mass sensitivity of the resonance frequency measurement is insensitive to the separation between cantilevers. Notice that the resonance frequency shifts are proportional to the total mass and are insensitive to how the mass is distributed on the cantilevers, whereas the amplitude ratio is proportional to the differential mass between the cantilevers. 

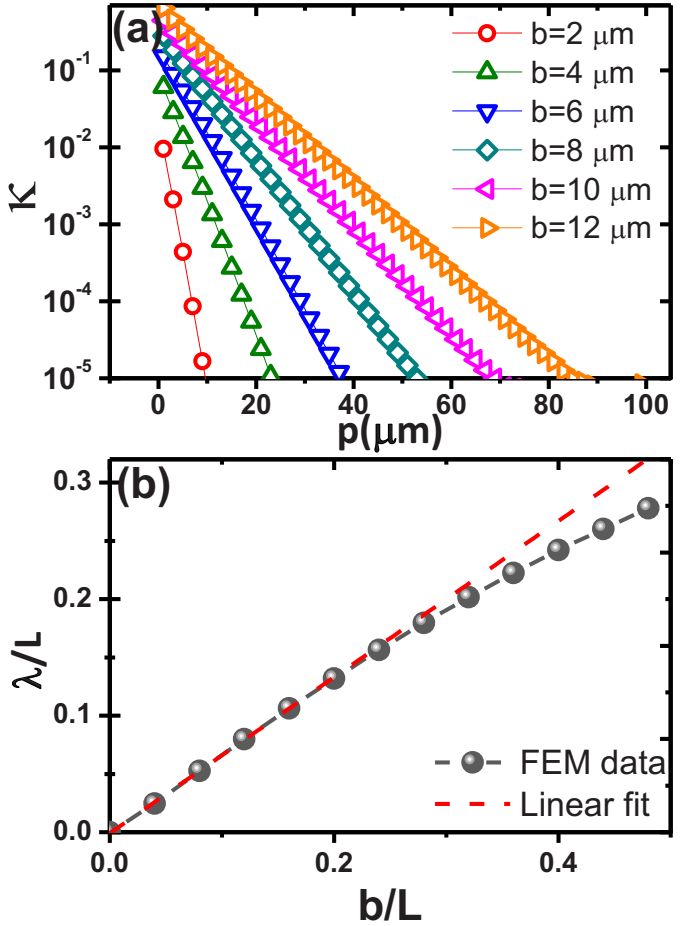

FIG. 4. (Color online) (a) Finite element simulation of the coupling constant (log scale) of a pair of coupled cantilevers such as those shown in Fig. 1(a) as a function of the gap separation, $p$, for different overhang lengths, $b$. The data clearly follows a simple exponential law A $e^{-p / \lambda}$. The decay constant distance, $\lambda$, is plotted vs the overhang length in (b). For overhang lengths $b<L / 4, \lambda \sim b$. The parameters $\lambda$ and $b$ have been normalized to the cantilever length $L$ that is $25 \mu \mathrm{m}$.

The combination of the harmonic oscillator theory for coupled systems and the fluctuation dissipation theorem provides, ${ }^{12}$

$$
a_{t h} \cong \frac{1}{\sqrt{1+2 \kappa}}\left[1 \pm(1+\kappa) \frac{\delta}{2 \kappa}\right],
$$

where $\delta$ is the ratio of the added mass to the cantilever effective mass. The positive sign stands for measurements in the cantilever in which the mass is not deposited, whereas the negative sign stands for the cantilever in which the mass is added. From Eq. (2) it is simple to derive that $\Delta a_{t h} / a_{t h}$ $\cong \delta / 2 \kappa$. Figure 3(c) shows the ratio of the change in vibration localization obtained by the measurement of $\Delta a_{t h} / a_{t h}$ to the eigenfrequency shift defined as $\Delta \omega_{a} / \omega_{a}+\Delta \omega_{s} / w_{s}$ for an added mass of about $100 \pm 10 \mathrm{fg}$ as a function of the coupling constant. This measurement allows to compare the vibration localization and resonance frequency methods. In addition, deviations in $\Delta a_{t h} / a_{t h}$ due to variations in the deposited mass are removed when it is divided by the relative frequency shift. The experimental data (symbols) show a good agreement with the theory (line) approximately given by $1 / 2 \kappa$. The amplitude ratio change is at least two orders of magnitude higher than the eigenfrequency shift for $\kappa<0.01$ that in our case corresponds to gaps larger than $20 \mu \mathrm{m}$. In addition, the mass sensitivity rapidly enhances as the coupling constant decreases.

Since the coupling constant plays a crucial role in the sensitivity of coupled nanomechanical systems, we have performed finite element simulations to determine how the cou- pling constant depends on the overhang length and width. Figure 4(a) shows $\kappa$ in logarithmic scale versus the gap distance $(p)$ for different overhang lengths $(b)$. The data shows that $\kappa$ exponentially decays with the gap size as found experimentally. The experimental results overlaps with the simulation data for $b=8 \pm 1 \mu \mathrm{m}$. We have fitted the simulation curves to $\kappa_{0} e^{-p / \lambda}$ and then we have plotted the exponential decay distance $\lambda$ versus the overhang length. For overhang lengths shorter than $L / 4, \lambda$ is linearly proportional to the overhang length. Therefore, $\kappa$ can be exponentially decreased by increasing the separation between the cantilevers or/and decreasing the overhang length.

To conclude, we have shown that the sensitivity of mass sensors based on the vibration localization phenomena in coupled cantilevers can be significantly enhanced by decreasing the coupling constant with no need of making the cantilevers smaller as it occurs when the mass is detected via the resonance frequency measurement. In particular, we have experimentally studied how the coupling constant of pairs of cantilevers elastically coupled by an overhang varies as a function of the separation between the cantilevers. The experiments show that the coupling constant exponentially decreases with the separation between cantilevers. In consistency with the theory the mass sensitivity is inversely proportional to the coupling constant. The exponential decay of the coupling constant with the cantilever separation is also found in finite element simulations. The simulations indicate that the coupling constant exponentially decreases with the ratio of the separation between cantilevers to the overhang length.

The authors acknowledge financial support from the Spanish Science Ministry through Project Nos. TEC200914517-C02, CSD2007-00010, and TRA2009-0117.

${ }^{1}$ T. Burg, M. Godin, S. Knudsen, W. Shen, G. Carlson, J. Foster, K. Babcock, and S. Manalis, Nature (London) 446, 1066 (2007).

${ }^{2}$ A. Naik, M. Hanay, W. Hiebert, X. Feng, and M. Roukes, Nat. Nanotechnol. 4, 445 (2009).

${ }^{3}$ T. Braun, M. Ghatkesar, N. Backmann, W. Grange, P. Boulanger, L. Letellier, H. Lang, A. Bietsch, C. Gerber, and M. Hegner, Nat. Nanotechnol. 4, 179 (2009).

${ }^{4}$ M. Varshney, P. Waggoner, C. Tan, K. Aubin, R. Montagna, and H. Craighead, Anal. Chem. 80, 2141 (2008).

${ }^{5}$ P. Waggoner and H. Craighead, Lab Chip 7, 1238 (2007).

${ }^{6}$ Y. Yang, C. Callegari, X. Feng, K. Ekinci, and M. Roukes, Nano Lett. 6, 583 (2006).

${ }^{7}$ K. Jensen, K. Kim, and A. Zettl, Nat. Nanotechnol. 3, 533 (2008).

${ }^{8}$ E. Gil-Santos, D. Ramos, J. Martínez, M. Fernández-Regúlez, R. García, Á. San Paulo, M. Calleja, and J. Tamayo, Nat. Nanotechnol. 5, 641 (2010).

${ }^{9}$ M. Spletzer, A. Raman, A. Wu, X. Xu, and R. Reifenberger, Appl. Phys. Lett. 88, 254102 (2006).

${ }^{10}$ M. Spletzer, A. Raman, H. Sumali, and J. Sullivan, Appl. Phys. Lett. 92, 114102 (2008).

${ }^{11}$ B. E. DeMartini, J. F. Rhoads, S. W. Shaw, and K. L. Turner, Sens. Actuators, A 137, 147 (2007).

${ }^{12}$ E. Gil-Santos, D. Ramos, A. Jana, M. Calleja, A. Raman, and J. Tamayo, Nano Lett. 9, 4122 (2009).

${ }^{13}$ P. Thiruvenkatanathan, J. Yan, J. Woodhouse, and A. Seshia, J. Microelectromech. Syst. 18, 1077 (2009).

${ }^{14}$ R. Karabalin, M. Cross, and M. Roukes, Phys. Rev. B 79, 165309 (2009)

${ }^{15}$ T. M. Huber, B. C. Abell, D. C. Mellema, M. Spletzer, and A. Raman, Appl. Phys. Lett. 97, 214101 (2010). 